\title{
MODELOS DE GESTÃO DA SEGURANÇA E SAÚDE NO TRABALHO: UMA REVISÃO SOBRE AS PRÁTICAS EXISTENTES E SUAS CARACTERÍSTICAS
}

\section{SAFETY AND HEALTH MANAGEMENT MODELS: EXISTENT PRATICES AND ITS CARACHTERISTICS - A REVIEW}

\author{
Luis Antonio dos Santos Franz ${ }^{1}$, Fernando Gonçalves Amaral ${ }^{2}$, Pedro Miguel Ferreira Martins \\ Arezes ${ }^{3}$. \\ ${ }^{1}$ Universidade Federal do Rio Grande do Sul - UFRGS - Porto Alegre - Brasil \\ luisantoniofranz@yahoo.com.br \\ ${ }^{2}$ Universidade Federal do Rio Grande do Sul - UFRGS - Porto Alegre - Brasil \\ amaral@producao.ufrgs.br \\ ${ }^{3}$ Universidade do Minho - UM - Guimarães - Portugal \\ parezes@dps.uminho.pt
}

\begin{abstract}
Resumo
A Segurança e Saúde no Trabalho (SST) são reconhecidas pela sua importância. Neste sentido, as perdas associadas a problemas nestas áreas apresentam amplas oportunidades de pesquisa. No presente trabalho fez-se a prospecção e análise na literatura dos recursos relacionados ao tema, indicando os princípios de base e sua abrangência. Buscou-se obter subsídios para a proposição de modelos que abordem não só ações para a implantação de sistemas de gestão da SST, mas também os meios para sua operacionalização. Verificou-se com o trabalho a existência de um foco demasiado em técnicas quando se pretende obter melhorias na área, o que por sua vez, induz à perda de visão sistêmica das questões associadas à segurança e saúde. Em contrapartida, observase em estudos mais recentes uma tendência à elaboração e uso de técnicas de atuação gerencial, bem como o crescimento de recursos que abrangem um maior número de etapas, no caso de implantação de um sistema de gestão da SST. O número de sistemas de gestão, contudo, ainda é baixo frente ao número de técnicas, o que indica a necessidade da elaboração de programas específicos para a gestão da SST.
\end{abstract}

Palavras-chave: segurança e saúde no trabalho, modelos de gestão

\section{Introdução}

Resultados proporcionados pela saúde e bem-estar dos trabalhadores são freqüentemente citados como fatores críticos de produtividade nas organizações, muito embora, sejam substituídos pelas necessidades de melhorias focadas em recursos mais específicos, como máquinas ou ferramentas. Em nível mundial, contudo, a Organização Internacional do Trabalho (OIT) estima que 
a cada ano, aproximadamente, três milhões de pessoas perdem suas vidas em decorrência de acidentes de trabalho, os quais chegam à taxa de 250 milhões ao ano (ILO, 2003). No Brasil, apenas no que se refere aos acidentes de trabalho, os números totalizaram no ano de 2005 uma cifra de 491.711 vítimas, acarretando em perdas financeiras expressivas (BRASIL, 2005). Neste contexto, os custos indiretos representados pela perda da produtividade e da produção, indenizações e compensações salariais, dentre outras, foram estimados em torno de 14,5 bilhões de dólares (BRASIL, 2005).

De fato, a dimensão das perdas associadas ao problema de segurança e saúde no trabalho é importante, e o campo de estudo nessa área apresenta amplas oportunidades de pesquisa. A segurança do trabalho está associada à interação entre as pessoas e suas atividades diárias, aos materiais, aos equipamentos e máquinas, ao meio ambiente e aos aspectos de produtividade. Para que isto seja possível, deve-se sempre buscar o equilíbrio entre o trabalho sadio e o nível de produtividade mais elevado possível (SKYBA, 1998). A busca por esse equilíbrio é a base dos estudos voltados à SST e também o princípio básico para a elaboração de aplicações na área. Em contrapartida, observa-se que nem as técnicas específicas, nem os sistemas de gestão ou avaliação existentes contemplam, ao mesmo tempo, as ações necessárias para atuar pontualmente e gerir a SST. Uma vez que, tais recursos fornecem separadamente apoios complementares, é possível supor que eles podem ser estratificados segundo determinados critérios como, por exemplo, pela sua abrangência, e posteriormente ser integrados.

Os sistemas de gestão da SST estão normalmente apoiados em políticas com uma visão generalista. Um exemplo de política é apresentado pela Organização Internacional do Trabalho (ILO-OSH 2001), que possui um espectro amplo de aplicações em organizações de diferentes nacionalidades. Porém, não contempla de forma detalhada as orientações necessárias para o estabelecimento de um programa ou sistema voltado à gestão da SST. Esse papel é assumido por normas mais específicas em cada país, como por exemplo: a OHSAS 18001, a norma BS 8800 no Reino Unido, ou ainda por sistemas de premiação como o MINEX (National Mineral Industry Excellence Award for Safety and Health) na Austrália (MCA, 2007).

Quando a abrangência é menor, não se tem mais os sistemas de gestão da SST, mas sim, técnicas específicas que tratam de temas pontuais. Alguns exemplos neste caso, são as técnicas voltadas à avaliação da confiabilidade humana, como THERP (Technique for Human Error Rate Prediction) e HEART (Human Cognition Reliability) (HOLLNAGEL, 1996; KIRWAN, 1996; 1997); ATHEANA (A Technique for Human Error Analysis) (COOPER et al., 1997); ou HCR (Human Cognition Reliability) (HOLLNAGEL, 1996).

Uma tendência recente, segundo Almeida (2006), é a abordagem sistêmica dos acidentes de trabalho, onde a visão culpabilística do acidente de trabalho é transferida para um contexto no qual 
o erro humano que, embora não deixe de ser considerado, passa a não ser a causa raiz dos acidentes e, mesmo a concepção de uma única causa raiz deixa de ser considerada. Dentre estas linhas de trabalho é possível citar o trabalho de Leveson (2004), que trata da problemática do erro humano dentro do contexto de acidentes. Porém, mesmo neste trabalho, a estruturação e os mecanismos necessários para uma adequada gestão da segurança, não estão voltados para uma modelo de gestão mais abrangente. Isso significa que a estrutura de tomada de ações, indicadores de desempenho ou considerações sobre o posicionamento estratégico da SST no organograma da empresa, não são abordados conjuntamente.

Por outro lado, a OHSAS 18001 e o modelo MINEX (MCA, 2007) são sistemas que apontam caminhos os quais as empresas devem seguir para alcançar um nível satisfatório na gestão da SST. Destes dois sistemas, a OHSAS é certamente o mais difundido, considerando-se que o MINEX é voltado exclusivamente para o setor de mineração australiano. Embora ambos contemplem todo ciclo de planejamento, implantação, verificação e ação, estes sistemas não esclarecem como atuar para alcançar os objetivos por eles apontados.

Dessa forma, observa-se que nem as técnicas específicas e nem os sistemas de gestão ou avaliação existentes contemplam, ao mesmo tempo, as ações necessárias para gerenciar e atuar pontualmente na SST. Embora, forneçam separadamente subsídios complementares, esta complementaridade permite supor que tais recursos podem ser estratificados segundo determinados critérios como, por exemplo, pela sua abrangência, e posteriormente integrados.

No que se refere ao estabelecimento dos critérios, a OHSAS 18001 sugere que durante a implantação de sistemas de gestão da SST deve-se atentar para: $a$ ) o planejamento; $b$ ) a implantação e operação; e c) a verificação e ações corretivas; sugerindo assim, que tais estratos são importantes na gestão da SST. Estes três blocos seguem, segundo a própria norma sugere, uma seqüência similar a do ciclo PDCA (DEMING, 1986), já amplamente difundido em organizações de diversos países. O MINEX, por sua vez, estabelece que um sistema de gestão da SST deve atentar para seis critérios: $a$ ) liderança; $b$ ) gestão da SST; $c$ ) pessoas; $d$ ) informação e análise; $e$ ) processos para a segurança e saúde no trabalho; e $f$ ) desempenho. Estes são divididos em sub-elementos, os quais são avaliados em termos de enfoque, aplicação, resultados e melhoria (BARREIROS, 2002; MCA, 2007). Ele consiste em uma premiação de reconhecimento dos processos de saúde e segurança do trabalho aplicado em indústrias de mineração australianas. Este recurso, que foi criado em 1995 e modificado no início de 2007, é considerado elemento-chave pelo Minerals Council of Australia (MCA), para a disseminação da cultura de segurança e redução de acidentes em empresas mineradoras. Ele permite, através de avaliações feitas por pessoas especializadas, determinar o nível de maturidade em que a organização se encontra no que se refere à gestão da SST (MCA, 2007). 
A estratificação por critérios de recursos em SST, também pode ser encontrada em trabalhos independentes de bases científicas. Um exemplo é o trabalho desenvolvido pela Organização Européia para a Segurança do Tráfego Aéreo (Eurocontrol), onde desde 2002, uma base de dados tem sido mantida por este órgão, contemplando em sua última versão em torno de 630 técnicas aplicáveis à promoção da segurança (EVERDIJ; et al., 2007). Neste trabalho, a estratificação é feita com relação: $a$ ) ao domínio de aplicação da técnica em termos de setor produtivo; $b$ ) ao domínio de aplicação da técnica em termos de quem é enfocado; $c$ ) os momentos de utilização em uma eventual implantação de um sistema de gestão de SST; e $d$ ) o tempo de existência da técnica. Embora seja um trabalho abrangente esta base trata, prioritariamente, de técnicas para avaliação de riscos, não privilegiando os sistemas de gestão.

Em termos de estratificação o trabalho de Everdij, et al. (2007) é mais abrangente, se comparado à OHSAS 18001, o que pode ser inferido examinando a quantidade de recursos prospectados (aproximadamente 630) e pelo número de versões produzidas, que totalizam sete. Contudo, a OHSAS pode se mostrar mais útil no presente trabalho, pois se encontra mais difundida no Brasil e, também por estar voltada à implantação e utilizar uma lógica de melhoria contínua, o que não é o foco do trabalho de Everdij, et al. (2007). O MINEX, por fim, possui o mecanismo de melhoria similar à OHSAS, bem como seus critérios contemplam os elementos necessários à gestão adequada da SST. Contudo, se comparado ao trabalho de Everdij, et al. (2007), o modelo utilizado pelo MINEX é muito mais restrito, já que foi elaborado e disseminado exclusivamente em empresas do seguimento de mineração da Austrália.

As questões abordadas permitem evidenciar a lacuna existente e passível de estudo, sobretudo no que se refere em como aplicar as soluções já existentes na literatura científica e normativa com foco em SST. Além disso, é observável a necessidade de estratificação dos recursos existentes com base em critérios pré-estabelecidos como, por exemplo, seu tempo de existência, sua abrangência, seu foco de atuação. Estes critérios poderiam ser extraídos das bases teóricas que suportam os sistemas de gestão existentes.

Neste contexto, o presente trabalho tem por objetivo prospectar e analisar na literatura os recursos relacionados com a gestão da SST, indicando em que princípios se baseiam e qual sua abrangência. Com isso, será possível obter subsídios para a proposição de modelos que abordem não só ações para a implantação de sistemas de gestão da SST, mas também os meios para sua operacionalização.

\section{Procedimentos metodológicos}

Inicialmente, realizou-se a prospecção de estudos dedicados à promoção da segurança e saúde no trabalho. A partir da leitura de Everdij, et al. (2007) e OHSAS 18001; foram estabelecidos 
os critérios utilizados para a estratificação dos modelos de gestão da SST quanto ao Tipo de Recurso, ao Tipo de Abordagem, à Abrangência, ao Tempo de Existência, à Associação Metodológica e aos Principais Nichos de Aplicação. Estes critérios foram subdivididos em critérios mais restritos, conforme pode ser observado no Quadro 1. Após isso, trabalhos foram analisados prospectados através de pesquisa em bases de dados de periódicos reconhecidos como referência na área e em websites de instituições dedicadas à promoção e físcalização da SST como, por exemplo, o Eurocontrol (European Organisation for the Safety of Air Navigation) e o MCA (Minerals Council of Australia). De forma complementar, e quando necessário, foram utilizados também livros dedicados à discussão dos recursos analisados. Os critérios foram estabelecidos a partir da OHSAS e complementados pelo trabalho de Everdij, et al. (2007), já que este é abrangente, apesar estar direcionado prioritariamente à União Européia e às técnicas de avaliação de riscos. Para tanto, os critérios utilizados em Everdij, et al. (2007) sofreram adequações em seus nomes, de forma a atender os objetivos do presente trabalho.

Quadro 1- Critérios utilizados na estratificação dos modelos de gestão da SST

\begin{tabular}{|c|c|c|c|}
\hline & \multicolumn{3}{|c|}{ Critérios } \\
\hline \multirow{4}{*}{1} & \multirow{4}{*}{ Tipo de Recurso } & \multicolumn{2}{|l|}{ Política de Gestão } \\
\hline & & \multicolumn{2}{|l|}{ Sistema de Gestão } \\
\hline & & \multicolumn{2}{|l|}{ Sistema de Avaliação } \\
\hline & & \multicolumn{2}{|l|}{ Técnica Específica } \\
\hline \multirow{5}{*}{2} & \multirow{5}{*}{ Tipo de Abordagem } & \multicolumn{2}{|l|}{ Qualitativa } \\
\hline & & \multicolumn{2}{|l|}{ Quantitativa } \\
\hline & & \multicolumn{2}{|l|}{ Performance humana } \\
\hline & & \multicolumn{2}{|l|}{ Equipamento } \\
\hline & & \multicolumn{2}{|l|}{ Sistemas } \\
\hline 3 & Tempo de Existência & \multicolumn{2}{|c|}{ Data de origem ou publicação mais antiga } \\
\hline \multirow{5}{*}{4} & \multirow{5}{*}{ Abrangência } & \multicolumn{2}{|l|}{ Equipamento } \\
\hline & & \multicolumn{2}{|l|}{ Software } \\
\hline & & \multicolumn{2}{|l|}{ Pessoas/Recursos Humanos } \\
\hline & & \multicolumn{2}{|l|}{ Procedimentos } \\
\hline & & \multicolumn{2}{|l|}{ Organização } \\
\hline \multirow{10}{*}{5} & \multirow{10}{*}{$\begin{array}{l}\text { Associação } \\
\text { metodológica }\end{array}$} & \multirow{4}{*}{ Planejamento } & Identificação, Avaliação e Controle de Riscos \\
\hline & & & Meios de acesso à da informação \\
\hline & & & Delineamento estratégico \\
\hline & & & Delineamento da gestão \\
\hline & & \multirow{4}{*}{ Implantação e Operação } & Estrutura e Responsabilidades \\
\hline & & & Treinamento e Mobilização \\
\hline & & & Recursos Físicos de Informação \\
\hline & & & Controle Operacional \\
\hline & & \multirow{2}{*}{$\begin{array}{l}\text { Verificação e Ação } \\
\text { Corretiva }\end{array}$} & Indicadores de Desempenho \\
\hline & & & Manutenção e Reciclagem \\
\hline
\end{tabular}


O primeiro critério de estratificação utilizado refere-se ao tipo de recurso identificado. $\mathrm{O}$ recurso pode ser caracterizado conforme explicitado a seguir:

a) Políticas de Gestão - foram classificados nesta categoria aqueles recursos que forneciam orientações abrangentes para a adequada gestão da segurança, sem maiores detalhes;

b) Sistemas de Gestão - entraram nesta categoria aqueles recursos que contemplam a estruturação organizacional necessária para a gestão da SST;

c) Sistema de Avaliação - aqui foram classificados os recursos que são prioritariamente dedicados à avaliação da SST nas empresas. Recursos como normas, que podem sofrer auditoria, não foram considerados neste caso, pois já estão contemplados como sistemas de gestão;

d) Técnica Específica - foram classificados aqui todos aqueles recursos restritos a ações pontuais em SST como, por exemplo, técnicas restritas à avaliação de risco.

No que se refere ao segundo critério, tipo de abordagem utilizada pelo recurso, o recursos foram estratificados conforme apresentado a seguir:

a) Qualitativa - foram considerados recursos com abordagens qualitativas aqueles em que o levantamento de informação, avaliação ou pontuação fossem estabelecidos a partir de grupos focados ou inferências pessoais;

b) Quantitativa - os recursos com abordagem quantitativa foram considerados como sendo aqueles onde o levantamento de informação, avaliação ou pontuação fossem estabelecidos pragmaticamente a partir de dados numérico e procedimentos matemáticos;

c) Performance Humana - neste caso, os recursos escolhido é aquele que utiliza os princípios de comportamentais ou psicológicos humanos como ponto de partida para ações em termos de gestão de SST;

d) Equipamentos - os recursos classificados sob este critério foram aquele em que utilizase os equipamentos, máquinas ou ferramentas de trabalho como instrumento de melhoria na gestão de SST;

e) Sistemas - aqui, os recursos classificados foram aqueles onde bases de dados ou softwares sustentam a gestão da informação e a avaliação em termos de gestão de SST.

No terceiro critério estratificou-se a idade do recurso analisado, sendo estabelecidas duas formas de identificar a origem. Em uma, a data foi identificada pela apresentação clara da origem referida no trabalho analisado. Outra opção utilizada foi estabelecer a data pela publicação mais antiga sobre o recurso. 
Em seguida, no quarto critério de estratificação, foi determinada a abrangência do recurso, ou seja, quais os elementos de uma organização onde o mesmo costuma ser aplicado para a realização de melhorias:

a) Equipamentos - os recursos classificados aqui são aqueles que atuam com ênfase em equipamentos, máquinas e ferramentas, objetivando melhorias em segurança;

b) Software - os recursos classificados aqui são aqueles com ênfase na melhoria em sistemas informatizados;

c) Pessoas/Recursos Humanos - neste critério foram classificados aqueles recursos com melhoria direcionadas para aspectos comportamentais e de mudança cultural;

d) Procedimentos - recursos que atuem em melhorias em procedimentos e processos foram classificados neste grupo;

e) Organização - quando as ações de melhoria em SST contemplavam a organização como um todo, agindo em nível estratégico, o recurso foi classificado neste critério.

Finalmente, o quinto critério concerne à contribuição do recurso em uma eventual implantação de um sistema de gestão da SST. Para tanto, avaliou-se a sua associação às etapas típicas de implantação, que passam pelo planejamento, pela implantação e operação e pela verificação e ações corretivas. Estas etapas ainda foram divididas em áreas, sendo que cada uma dessas áreas funcionaram como os critérios a serem pontuados. Caso o recurso não contemplasse o critério em questão, atribuía-se o valor 0 . Se o recurso contemplasse superficialmente ou apenas fizesse referência, então era atribuído o valor 1. Quando contemplasse parcialmente e ainda sugerisse soluções, mas não os meios para aplicá-las, pontuava-se o valor 2. Por fim, se o recurso contemplasse em profundidade o critério em questão e ainda discutisse os meios para atendê-lo, então se atribuía o valor 3. Os valores atribuídos ficaram dispostos em uma matriz, conforme pode ser verificado de forma genérica no Quadro 2.

Para a avaliação da aderência dos recursos estudados a um sistema de gestão da SST, foram realizadas duas avaliações. Nas colunas, avaliou-se o quanto cada etapa era contemplada pelo recurso em questão, conforme a Equação (1). Para tanto, realizou-se o produto do número de vezes em que a etapa teve alguma pontuação acima de 0 pela soma das pontuações presentes na mesma. Nas linhas da matriz avaliou-se qual o recurso mais propício a ser utilizado considerando-se a implantação de um sistema de gestão da SST. O cálculo consistiu no produto do número de vezes em que o recurso teve alguma pontuação acima de 0 pela soma das pontuações presentes na linha. Quando o recurso analisado consistia em um sistema de gestão (SG) ou política de gestão (PO), atribuiu-se um peso de 1,5 ao valor final da operação matemática, conforme pode ser observado na Equação (2). A atribuição deste peso visa aumentar a importância dos recursos que eventualmente possam contribuir na proposição de modelos de gestão da SST. 


$$
\begin{aligned}
& \text { Quadro 2 - Matriz para avaliação de aderência } \\
& \begin{array}{|c|c|c|c|c|c|}
\hline \mathbf{C}_{\mathbf{1}} & \mathbf{C}_{\mathbf{2}} & \mathbf{C}_{\mathbf{3}} & \ldots & \mathbf{C}_{\mathbf{n}} & \\
\hline \mathrm{A}_{11} & \mathrm{~A}_{12} & \mathrm{~A}_{13} & \ldots & \mathrm{A}_{1 \mathrm{n}} & \mathbf{L}_{\mathbf{1}} \\
\hline \mathrm{A}_{21} & \mathrm{~A}_{22} & \mathrm{~A}_{23} & \ldots & \mathrm{A}_{2 \mathrm{n}} & \mathbf{L}_{\mathbf{2}} \\
\hline \ldots & \ldots & \ldots & & \ldots & \ldots \\
\hline \mathrm{A}_{\mathrm{m} 1} & \mathrm{~A}_{\mathrm{m} 2} & \mathrm{~A}_{\mathrm{m} 3} & \ldots & \mathrm{A}_{\mathrm{mn}} & \mathbf{L}_{\mathbf{m}} \\
\hline(1) & \mathrm{C}_{j}=m \sum_{i=1}^{m} A i j \\
\text { (2) } & L_{i}=1,5 n \sum_{j=1}^{n} A i j
\end{array} \\
&
\end{aligned}
$$

Além dos critérios citados, também foram levantadas informações complementares sobre os nichos de negócio em que os recursos são comumente aplicados ou têm, pelo menos, sua aplicação sugerida.

\section{Resultados}

Foram analisadas 51 referências. Os recursos levantados totalizaram 109 itens. Destes, 33 foram analisados em todos os critérios. Outros 76 recursos não tiveram um ou mais critérios avaliados quando: $a$ ) tratava-se de técnica específica; $b$ ) possuísse mais de 10 anos de existência; e c) apresentava baixa freqüência de ocorrência em bases de dados científicas.

Observando a bibliografia pesquisada, percebe-se que os recursos disponíveis são predominantemente técnicas específicas (72\%). Em seguida, mas com um percentual bem menor de ocorrência, encontram-se recursos voltados aos sistemas de gestão da SST (18\%), políticas de gestão da SST (6\%) e sistemas de avaliação (4\%). Assim, fica evidenciado que os trabalhos voltados à SST estão predominantemente focados em ações pontuais em termos de segurança, desprestigiando a importância dos sistemas de gestão para a promoção da SST.

Já, no que se refere ao tipo de abordagem utilizada, na maioria dos casos estudados é qualitativa (53\%). Isso pode mostrar, por um lado, a característica intrínseca da SST que exige um julgamento e tratamento mais subjetivo. Em outro sentido, pode apontar para a falta de rigor matemático e estatístico no tratamento das informações, o que é crítico, caso leve-se em conta a necessidade do uso de indicadores de desempenho. Quando ambos os tipos de abordagem são consideradas conjuntamente, pode-se perceber que tanto recursos qualitativos quanto quantitativos usam predominantemente abordagem em performance humana (ver Figura 1). Essa tendência observada não é necessariamente a mais apropriada, dado que a maior parte dos recursos existentes são técnicas especificas, ou seja, caracterizam-se por ações pontuais, exatamente onde seria 
esperado uso de recursos quantitativos.

Figura 1 - Tipo de abordagem utilizada pelo recurso

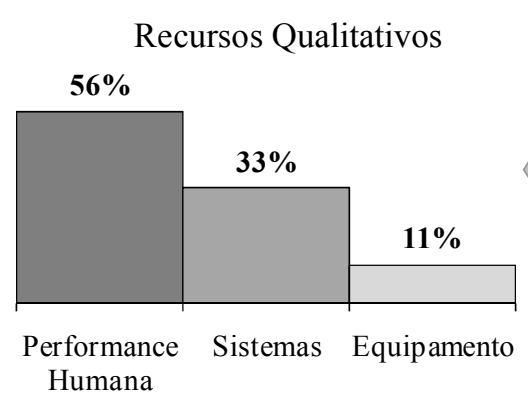

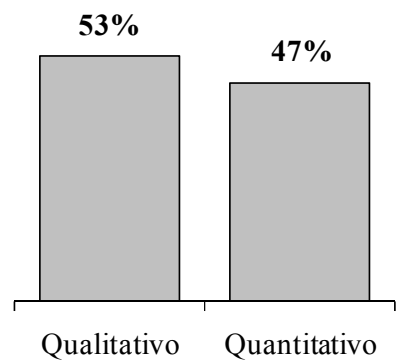

Fonte: Autoria própria (2008)
Recursos Quantitativos $62 \%$

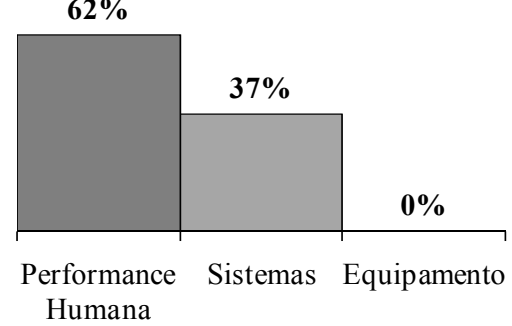

Conforme pode ser observado na 0 , o número de recursos voltados à SST aumentou a uma taxa quase constante no decorrer das últimas décadas. Pode-se inferir, pelo comportamento do gráfico, que houve uma desaceleração no número de novos recursos, dado a data mais recente de 2007.

Figura 2 - Períodos de origem dos recursos estudados

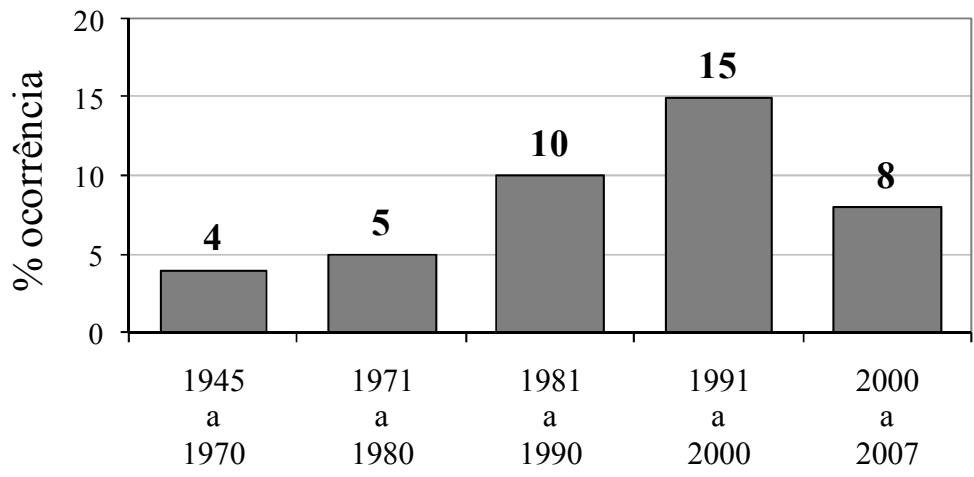

Data de origem dos recursos

Fonte: Autoria própria (2008)

Os valores apresentados no gráfico da Figura 3 foram obtidos a partir da Equação (1). Por estes, é possível constatar que a maior parte dos recursos contempla as atividades de verificação e ação corretiva nos sistemas de SST. Ou seja, os aspectos relacionados ao planejamento dos sistemas de gestão da SST e seu alinhamento às estratégias organizacionais não são focados pela maior parte dos recursos. Mesmo as técnicas específicas, que têm maior ênfase na avaliação e manutenção dos programas, não estão, em sua maioria, associadas à implantação dos sistemas de gestão. 
Figura 3 - Associação dos recursos às etapas de implantação de um sistema de gestão de SST

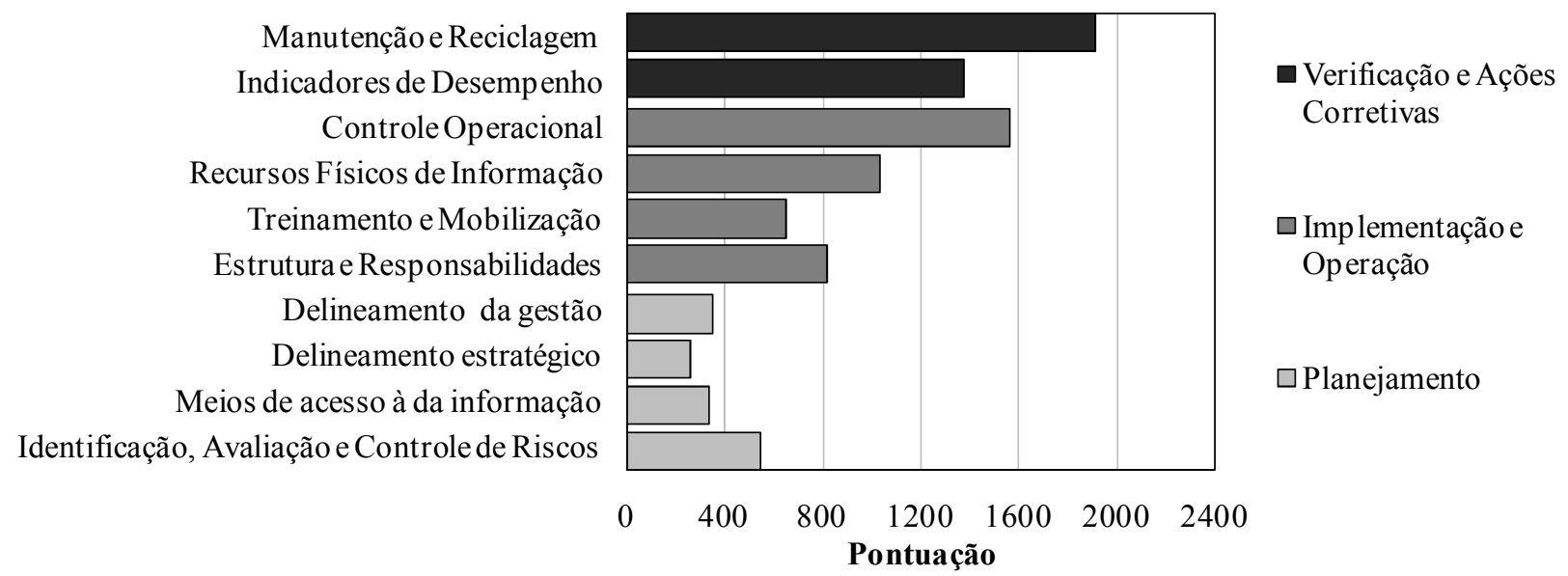

Fonte: Autoria própria (2008)

Ao utilizar os procedimentos matemáticos a partir da Equação (2), foi possível obter o gráfico apresentado na Figura 4. Pelo mesmo, é possível inferir que, dentre os 20\% dos itens com maior pontuação (12 recursos), pelo menos três são bastante semelhantes (OHSAS 18001, BS 8800 e OIT). Dois são usados em conjunto, a estratégia SOBANE e o DEPARIS. Entre os demais itens com maior pontuação, pode-se citar o caso do MINEX e do ASSET, que embora não contemplem de forma tão eficiente todos os aspectos de um sistema de gestão da SST, podem contribuir em termos de aplicação pela simplicidade de utilização. O INDICATE (Identifying Needed Defences In The Civil Aviation Transport Environment), de acordo com Edkins (1998), aponta para ações em gestão da SST. Neste recurso é possível encontrar orientações quanto ao papel da gerência e suas responsabilidades, bem como algumas ações necessárias para a disseminação da informação relativa à segurança em todos os níveis organizacionais.

Figura 4 - Itens priorizados com base na associação dos recursos às etapas de um sistema de gestão da SST

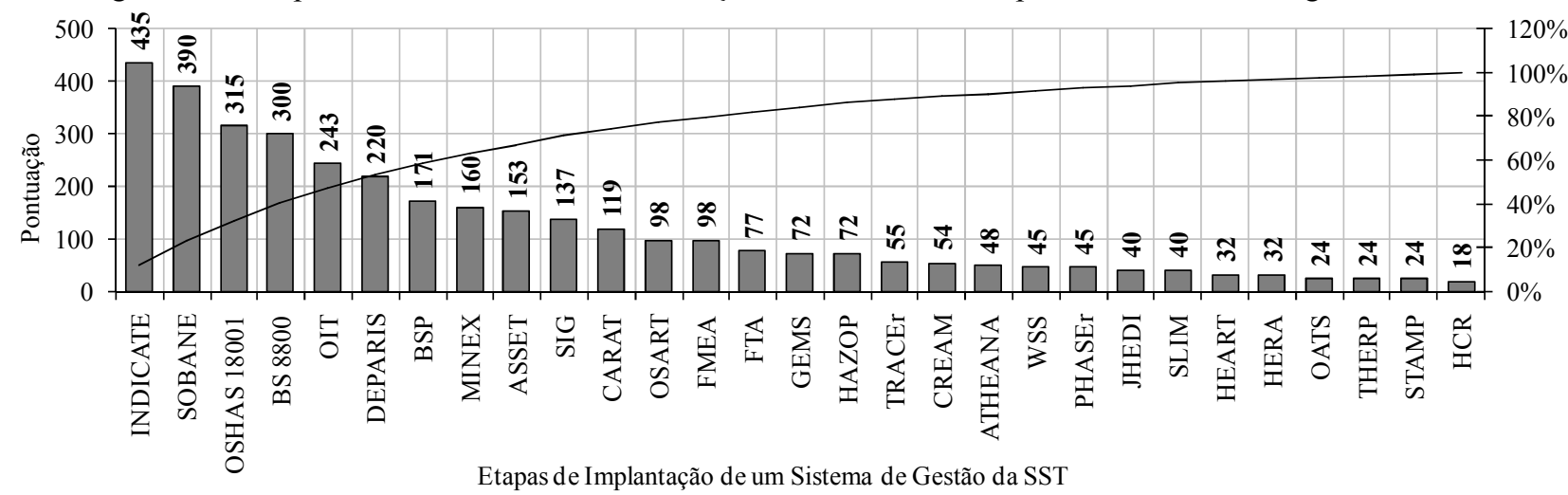

Fonte: Autoria própria (2008)

No tocante à abrangência, os recursos em sua maioria (36\%), abrangem melhorias em recursos humanos. Esse resultado tem relação com o comportamento verificado nas abordagens utilizadas, onde os recursos mostram-se predominantemente qualitativos e sustentados em 
princípios psicológicos e comportamentais. Quanto aos principais nichos de negócio abrangidos pelos recursos, a análise mostra que com grande freqüência (40\%) estes se originaram ou foram aplicados primeiramente, em organizações que trabalham com energia nuclear. Ao somar o percentual englobando indústria aeroespacial e química, estas taxas ampliam-se para 73\%. Isto significa que tais áreas são as principais promotoras de ações e de pesquisas, objetivando melhorias na segurança e saúde do trabalho.

Ao cruzar os dados percebe-se que os recursos identificados como sistemas de gestão contemplam maior abrangência de ações em SST. Todos eles utilizam abordagem qualitativa e têm foco em melhorias no nível organizacional. Um recurso atípico é o ASSET (SWANSON et al., 2004), que possui característica de ferramenta e é apoiado em um software robusto. Este recurso, contudo, também se enquadra como um sistema de gestão, pelo fato de estar amparado em um sistema informatizado que lhe permite ser amplamente disseminado pela organização fornecendo, inclusive, subsídios para o uso de indicadores de desempenho. Outro diferencial, frente aos demais recursos, é sua forma de tratamento dos dados, o que lhe confere um status de abordagem qualitativa.

Ainda, em termos de sistema de gestão, os recursos que parecem estar mais alinhados ao planejamento é o modelo SOBANE (MALCHAIRE, 2001; 2002; MALCHAIRE; PIETTE, 2006) e o modelo INDICATE (ATSB, 2001; EDKINS, 1998). Eles contemplam em profundidade aspectos do delineamento estratégico da gestão da segurança, sendo que o segundo propõe ainda um software que ampara sua implantação e gerenciamento. Contudo, em ambos os casos não é observada nenhuma referência quanto ao alinhamento destes às diretrizes estratégicas organizacionais.

As políticas de gestão e sistemas de avaliação estão bem menos presentes nas referências dedicadas à gestão da segurança. Uma causa provável é o fato da OIT se caracterizar como documento de referência frente aos demais recursos dedicados ao tema, eliminando a necessidade de outros trabalhos com este enfoque. A norma OHSAS 18001 também contempla as políticas de segurança, porém de forma mais resumida que a OIT (ILO, 2001).

Em termos de sistemas de avaliação foram identificados dois recursos, um dedicado ao setor de mineração e outro à energia nuclear. Contudo, só o primeiro, MINEX (National Mineral Industry Excellence Award for Safety and Health), demonstra tal característica, inclusive apontando uma grade de pontuação, similar aos modelos utilizados para avaliação de maturidade. Em contrapartida, o segundo, denominado OSART (Operational Adivisory Safety Review Team), é em sua essência um modelo avaliação, sendo que neste caso (TAYLOR, 1997; BAUMONT, 2000; LIPÁR, 2003) enfatizam não ser este o objetivo do recurso. A OHSAS 18001 e BS 8800, embora possam se utilizar de auditorias propositalmente este não seu foco principal, logo não foram 
considerados como sistemas de avaliação no presente trabalho.

Observando-se o tempo de existência dos recursos analisados é possível notar que suas abordagens mudaram no decorrer de épocas mais recentes. Percebe-se pelo gráfico da Figura 5 que nas últimas três décadas houve um crescimento expressivo de recursos centrados em melhorias em nível organizacional. No mesmo período, a quantidade de recursos com foco em pessoas diminuiu e recursos focados em procedimentos e equipamentos mantiveram-se estáveis. Ao mesmo tempo, a Figura 6 apresenta um crescimento constante das abordagens qualitativas. A combinação destes dois comportamentos mostra uma nova tendência, onde possivelmente o foco dos recursos tem se direcionado para a ênfase especifica em mudança cultural, utilizando para isso abordagens qualitativas e atuando em níveis gerenciais mais altos.

Figura 5 - Distribuição dos recursos para melhorias em SST considerando seu foco

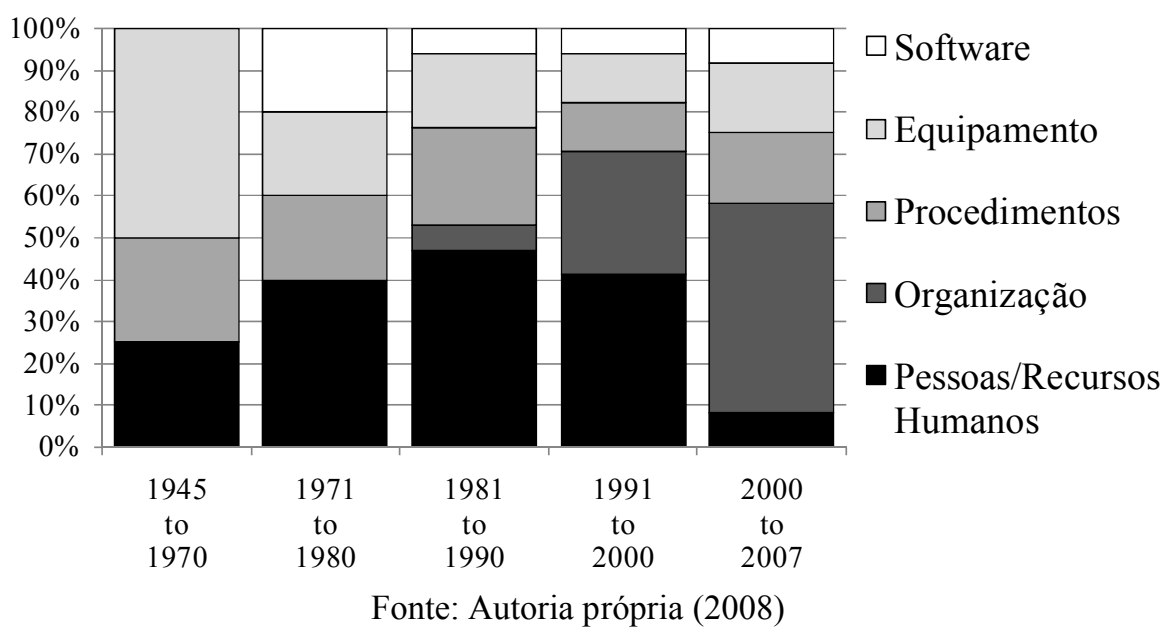

Figura 6 - Distribuição dos recursos para melhorias em SST considerando o tipo de abordagem

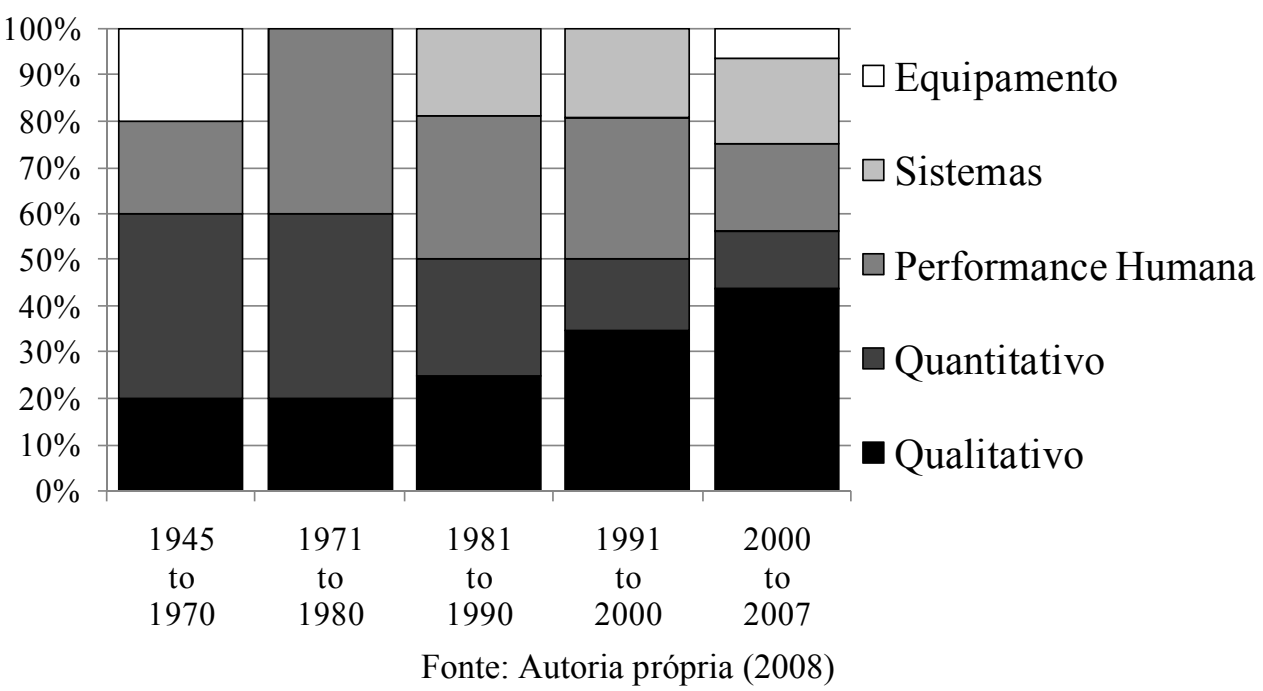

Nota-se pelo gráfico da Figura 7 que, somente em meados da década de 80, os recursos passaram a se dedicar com maior ênfase à etapa de planejamento dos sistemas de gestão da SST. 
Atualmente, há um maior equilíbrio entre planejamento, implantação e manutenção dos sistemas de gestão da SST.

Figura 7 - Comportamento da aderência dos recursos a um sistema de gestão da SST

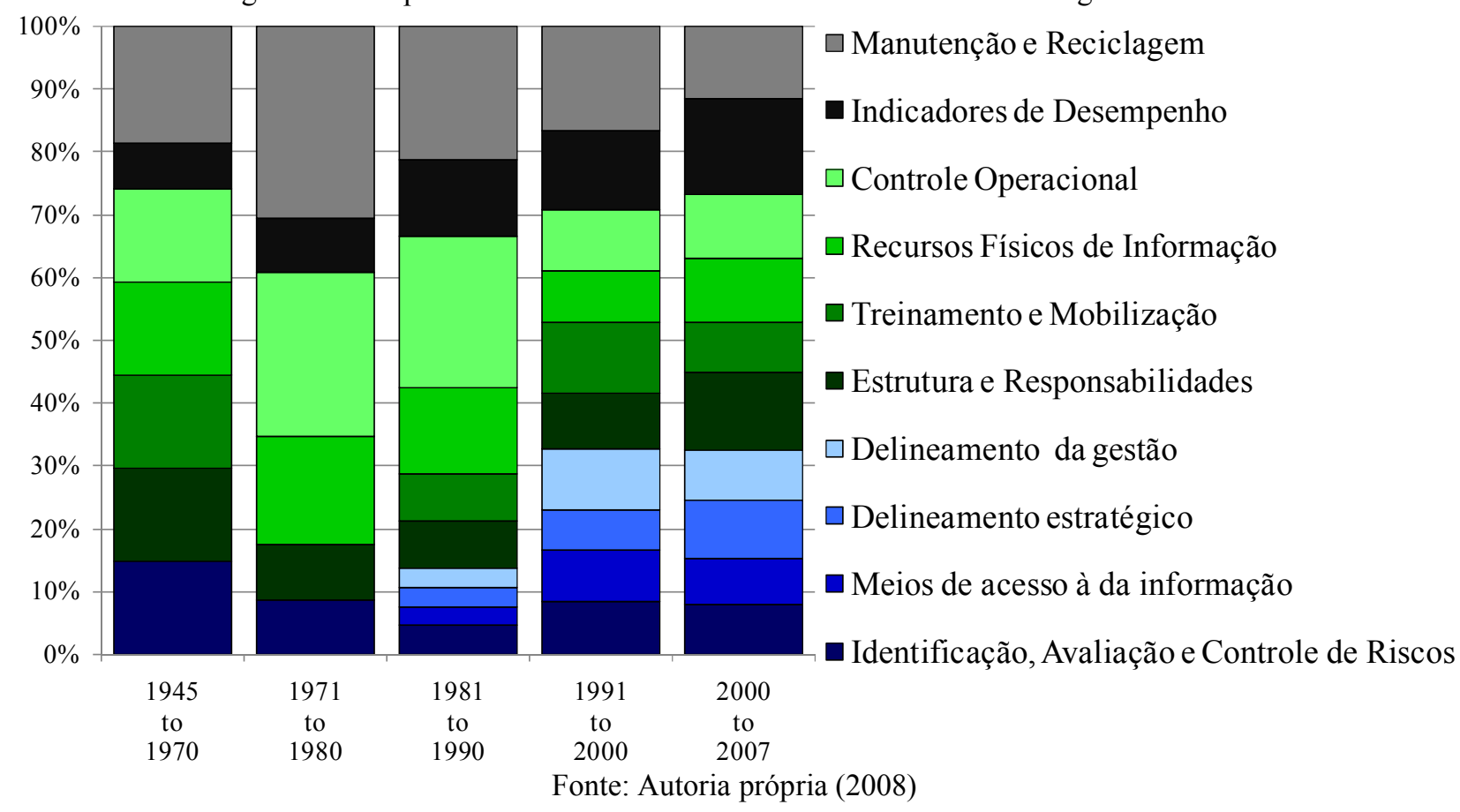

\section{Discussão e Conclusão}

As evidências encontradas, através da pesquisa realizada, apontam para uma excessiva centralização na aplicação de técnicas quando se pretende obter melhorias em SST. Embora a existência de recursos deste tipo possa se mostrar especialmente útil, o seu uso exclusivo tende a não funcionar como impulsionador de mudança cultural nas organizações. Mudanças mais profundas em SST, de forma similar ao que acontece com a gestão da qualidade, precisam de iniciativas mais abrangentes e que contemplem mudanças em níveis gerenciais mais altos. Em programas de gestão da qualidade, autores como Pande, et al. (2001), sugerem que todos os níveis gerenciais precisam estar comprometidos com a iniciativa para que o programa tenha sucesso.

Outro fator decorrente da prevalência de técnicas em detrimento de sistemas de gestão é a perda de visão sistêmica na gestão da SST. Quando as causas e efeitos em SST são tratados pontualmente, perde-se informações que poderiam amparar melhorias mais eficientes. Desta forma, tanto o planejamento estratégico, a medicina do trabalho ou as equipes de segurança deveriam atuar de forma integrada em termos de indicadores de desempenho e tomada de decisão, caso contrário ações de melhoria em segurança se tornam naturalmente ineficientes. Ainda, faz-se necessário que a cultura de segurança permeie todos os níveis organizacionais.

Há um comportamento que chama a atenção nos dados obtidos é o crescimento na quantidade de técnicas específicas disponíveis, acompanhado da queda da abordagem em 
performance humana, bem como a queda do foco das melhorias em pessoas. Por outro lado, o aumento em abordagens qualitativas e em melhorias com abrangência organizacional leva à possibilidade de que as técnicas vêm sendo elaboradas de forma a interagir mais intimamente com os níveis gerenciais mais altos. É consenso que a cultura de SST deve estar difundida igualmente em todos os níveis de uma empresa, contudo o próprio número elevado de técnicas mostra que muito ainda necessita ser realizado nesse sentido. As diretrizes de modelos de gestão como INDICATE (ATSB, 2001), SOBANE (MALCHAIRE, 2001) e OHSAS 18001, são exemplos de modelos que apontam eminentemente para este aspecto.

Apesar dos sistemas de gestão da SST terem ganhado importância frente às técnicas nas últimas décadas, conforme pode ser observado na Erro! Fonte de referência não encontrada. e na 0, ainda é incipiente a utilização dos mesmos. No Brasil, por exemplo, enquanto a NBR ISO 9001 apresenta um total acumulado de aproximadamente 23.000 certificações desde 1990, a OHSAS, segundo dados fornecidos pela Revista Proteção, em 2006 ficava em torno 400 certificações (PROTEÇÃO, 2006). Cabe ressaltar que, mesmo não se caracterizando como técnica específica, a OHSAS 18001 possui a abordagem descritiva, que define o que deve ser feito, mas não esclarece como fazê-lo. Essa característica é uma herança das séries ISO e BS, que a influenciam de forma importante.

Cada organização possui suas características próprias. Contudo, de forma similar ao que acontece com gestão da qualidade, os programas de gestão da SST poderiam orientar sobre como realizar e manter as melhorias pretendidas. Um exemplo é o INDICATE (Identifying Needed Defenses In The Civil Aviation Transport Environment) (ATSB, 2001), o qual apresenta uma descrição detalhada de como proceder na implantação de um sistema de gestão da SST, mas que não aponta soluções para a integração da segurança e saúde no trabalho com a estratégia organizacional.

Verificou-se que atualmente as técnicas específicas associam-se principalmente às áreas de manutenção e avaliação do programas de gestão, não sendo comumente associadas à implantação destes, situação que poderia ser revertida caso houvesse uma disseminação maior dos sistemas de gestão, ao invés do surgimento de novas técnicas. Caso contrário, a ineficiência para transformar ações em melhorias efetivas em SST ainda continuará sendo uma situação recorrente nas empresas. Essa reversão poderia ser motivada por programas dedicados a tomar ações de melhoria em nível organizacional, nos moldes do que acontece com os programas de qualidade. Recursos típicos da gestão da qualidade, como a metodologia Seis Sigma (PANDE; et al. 2001) poderiam auxiliar no sentido de oferecer subsídios para a elaboração de um sistema gestão da SST mais eficiente. Abordagens sobre o uso conjunto de conceitos de segurança e Seis Sigma podem ser verificados em Revelle (2004) e Rancour e McCracken (2000). 
Por fim, o estudo da associação dos recursos às áreas típicas de um sistema de gestão da SST, sugere que recursos como a estratégia SOBANE, o DEPARIS ou o INDICATE, podem amparar de forma mais abrangente a implantação de sistemas de gestão da SST. Como complemento ao presente estudo, sugere-se a realização de pesquisas para aprofundar o levantamento de recursos que dão apoio à segurança e saúde no trabalho e que poderiam ser aplicados em conjunto com os modelos tipicamente utilizados na gestão da qualidade.

\begin{abstract}
The Safety and Health at Work (SHW) are recognized by his importance. In this sense, the losses associated to problems in these areas present wide research opportunities. In the present work it was made the search and analysis in the literature of the resources related to theme, indicating the base beginnings and her inclusion. It was tried to obtain subsidies for the proposition of models that its approach not only actions for the implantation of systems of administration of SHW, but also the means for implementation. It was verified the existence of a too much focus in techniques when it intend to obtain improvements in the area. It induces to the loss of systemic vision of the subjects associated to the safety and health. On other hand, it is observed in more recent studies a tendency to the elaboration and use of techniques of managerial approach, as well as the growth of resources that its include a larger number of stages, in the case of implantation of a system of administration of SHW. The number of administration systems, however, it is still low front to the number of techniques. This indicates the need of the elaboration of specific programs for the administration of SHW.
\end{abstract}

Key-words: Safety and health at work, administration models

\title{
Referências
}

ALMEIDA, I. M. Abordagem sistêmica de acidentes e sistemas de gestão de saúde e segurança do trabalho. INTERFACEHS - Revista de Gestão Integrada em Saúde do Trabalho e Meio Ambiente, vol. 1, n. 2, disponível em: $<\mathrm{http}: / /$ www.interfacehs.sp.senac.br/br/artigos.asp?ed=2\&cod_artigo=32>. Acesso em: 19 mar. 2006.

ATSB. The INDICATE safety program: implementation guide. ASTB - Australian Transport Safety Bureau, v 2.0, 2001.

BARREIROS, D. Gestão da segurança e saúde no trabalho: estudo de um modelo sistêmico para as organizações do setor mineral. Tese de Doutorado: Escola Politécnica da Universidade de São Paulo. Departamento de Engenharia de Minas e Petróleo - USP, 2002.

BAUMONT, G.; et al. Organizational factors. Their definition and influence on nuclear safety. Final report. Espoo 2000. Technical Research Centre of Finland, VTT Tiedotteita - Meddelanden - Research notes 2067, 2000.

BRASIL. Anuário Estatístico da Previdência Social/Ministério da Previdência. Brasília: MPS/DATAPREV, v.14, p.1-834, 2005.

BRITISH STANDARDS INSTITUTION. BS 8800: guide to occupational health and safety management systems. British Standards Institution, London, 1996.

BRITISH STANDARDS INSTITUTION. OHSAS 18001: occupational health and safety management systems specification. British Standards Institution, London, 1999.

COOPER, S. E.; et al. The Application of ATHEANA: A Technique for Human Error Analysis. IEEE sixth annual human factors meeting. vol. 9, p. 13-17, 1997.

DEMING, W. E. Out of the Crisis. Massachusetts: Mit Caes, 7 ed., 1986. 
EDKINS, G. D. The Indicate safety program: evaluation of a method to proactively improve airline safety performance. Safety science, n 30, p 275-295, 1998.

cross'r

EVERDIJ, M. H. C.; et al. Development of a structured database of safety methods. 2007.

HOLLNAGEL, E. Reliability analysis and operator modeling. Reliability Engineering and System Safety. vol. 52, p. 327-337, 1996.

cross ${ }^{\text {ref }}$

ILO - International Labour Organization. Safety in numbers: pointers for the global safety at work. Geneva; 2003.

ILO-OSH 2001. Directrices relativas a los sistemas de gestión de la seguridad y la salud em el trabajo. Oficina Internacional del Trabajo. Genebra. 2001.

KIRWAN, B. The validation of three Human Reliability Quantification techniques - THERP, HEART and JHEDI: Part I - Technique descriptions and validation issues. Applied Ergonomics. vol. 27, n. 6, p. 359-373, 1996.

cross ${ }^{\text {ref }}$

KIRWAN, B. The validation of three Human Reliability Quantification techniques - THERP, HEART and JHEDI: Part II - Practical aspects of the usage of the techniques. Applied Ergonomics. vol. 28, n. 1, p. 27-39, 1997.

cross'

LEVESON, N. G. A new accident model for Engineering Safer Systems. Safety Science, v.42, p.237-70, 2004.

cross ${ }^{\text {ref }}$

LIPÁR, M. Status and trends in IAEA safety standards. In: International ILK - Symposium 2003, Munich, Vienna, 2003.

MALCHAIRE, J., et al. Évaluation du risque de contrainte thermique lors du travail en ambiances chaudes. Médecine du Travail \& Ergonomie, Volume XXXVIII, Nº 3, 2001.

MALCHAIRE, J.. DÉpistage PArticipatif des RISques dans une situation de travail: Méthode DEPARIS. Volume XXXIX, N $4,2002$.

MALCHAIRE, J.; PIETTE, A. The SOBANE Strategy for the Management of Risk, as Applied to Whole-Body or Hand-Arm Vibration. Annals Occupational Hygiene, vol. 50, N. 4, p. 411-416, 2006.

cross ${ }^{\text {ref }}$

MCA. MINEX 2007: Minerals industry safety and health excellence awards background, assessment criteria and evaluation process. Minerals Council of Australia: Safety \& Health. Disponível em: $<$ http://www.minerals.org.au/safety/minex/minex>. Acesso em: 15 abr. 2007.

PANDE, P. S.; et al. Estratégia Seis Sigma: como a GE, a Motorola e outras grandes empresas estão aguçando seu desempenho. 1 ed. Rio de Janeiro: Qualitymark. 2001.

PROTEÇÃO. Anuário brasileiro de proteção 2006. Revista Proteção. Disponível em: $<$ http://www.protecao.com.br/novo/template/page.asp?menu=575\&CodMenu=575\&Lbt=0 $>$. Acesso em: 09 out. 2006.

RANCOUR, T.; McCRACKEN, M. Applying 6 sigma methods for breakthrough safety performance. Professional Safety. v. 45, n. 10, p. 29-32, oct 2000.

REVELLE, J. B. Six Sigma: Problem-solving techniques create safer, healthier worksites. Professional Safety, vol. 49, n. 10 , p 38-46, out 2004 .

SWANSON, M.; et al. Automated Security Self-Evaluation Tool User Manual. National Institute of Standards and Technology. In:< http://csrc.nist.gov/archive/asset/Version2/ASSET_User_Manual_2004ED.pdf >, acessado em: 15/03/2007, 16h00. 2004.

TAYLOR, R. B. Operational safety review of nuclear power plants: lecture notes for the course on. 1997. 


\section{Inserir aqui dados completos de TODOS os autores:}

Nome completo: Luis Antonio dos Santos Franz

Filiação institucional: Universidade Federal do Rio Grande do Sul

Departamento: Engenharia de Produção e Transportes

Função ou cargo ocupado: Doutorando

Endereço completo para correspondência (bairro, cidade, estado, país e CEP):

Av. Osvaldo Aranha, 99 - 5 ${ }^{\circ}$ andar, centro, Porto Alegre, RS, Brasil, 90035-190

Telefones para contato: (51) 3308.4292

e-mail: luisantoniofranz@yahoo.com.br

Nome completo: Fernando Gonçalves Amaral

Filiação institucional: Universidade Federal do Rio Grande do Sul

Departamento: Engenharia de Produção e Transportes

Função ou cargo ocupado: professor Adjunto

Endereço completo para correspondência (bairro, cidade, estado, país e CEP):

Av. Osvaldo Aranha, 99 - $5^{\circ}$ andar, centro, Porto Alegre, RS, Brasil, 90035-190

Telefones para contato: (51) 3308.4292 - (51) 9967.1234

e-mail: amaral@producao.ufrgs.br

Nome completo: Pedro Miguel Ferreira Martins Arezes

Filiação institucional: Universidado Minho

Departamento: Departamento de Produção e Sistemas

Função ou cargo ocupado: professor Adjunto

Endereço completo para correspondência (bairro, cidade, estado, país e CEP):

Universidade do Minho - Campus de Azurem - 4800-058 - Escola de Engenharia - Departamento de Produção e Sistemas

Telefones para contato: +351253510340 - +351253510354

e-mail: parezes@dps.uminho.pt 